\title{
Planar arrayed waveguide device structure for 2D wavelength demultiplexing
}

\author{
Hideaki Okayama ${ }^{\text {a) }}$ \\ R\&D Group, Oki Electric Industry Co., Ltd., 550-5 Higashiasakawa-cho, Hachioji, \\ Tokyo 193-8550, Japan \\ RISE, Waseda University, 3-1-4 Okubo, Shinjyuku, Tokyo, 153-8555, Japan \\ a)okayama575@oki.com
}

Abstract: Simple arrayed waveguide grating (AWG) structure which demultiplexes wavelengths to output ports arranged in 2D plane is proposed. The planar geometry provides easy fabrication of the device. An AWG is connected to a device forming a 2D aperture array, which generates smaller dispersion in orthogonal direction against that generated by AWG.

Keywords: arrayed waveguide grating, wavelength demultiplexer, virtual image, waveguide mirror

Classification: Photonics devices, circuits, and systems

\section{References}

[1] K. Furuhashi, H. Okayama, and H. Nakajima, Tech. Digest OECC2003, (Shanghai, China) Paper pp. 2-15.

[2] J. Yang, X. Jiang, M. Wang, and Y. Wang, "Two-dimensional wavelength demultiplexing employing multilevel arrayed waveguides," Opt. Express, vol. 12, no. 6, p. 1084, 2004.

[3] H. Okayama, Tech. Digest CPT2004, (Tokyo, Japan) Paper p. 14.

[4] M. K. Smit and C. Van Dam, "PHASAR-based WDM-devices: Principles, design, and applications," IEEE J. Select. Topics Quantum Electron., vol. 2, p. 236, June 1996.

[5] S. Tang, M. M. Li, and R. T. Chen, IEEE Photon. Tech. Lett., vol. 7, p. $908,1995$.

[6] C. Moser, F. Havermeyer, W. Liu, G. Steckman, K. Buse, and D. Psaltis, Tech. Digest OFC2003, (Atlanta, USA) Paper FC6.

\section{Introduction}

Many types of wavelength demultiplexers have been proposed for WDM optical communication and other applications such as wavelength monitoring. Most of the devices separate wavelengths into output ports arranged in a line (1D demultiplexing). When we can demultiplex the wavelengths into output ports arranged in two-dimensional (2D) plane, the number of ports could be increased dramatically. Device configurations using two cascaded wavelength 
dependent light beam steering devices each assigned for orthogonal spatial axis ( $\mathrm{x}$ and $\mathrm{y}$ axis) have been known for some time. Recently we proposed a method using only a single device to realize $2 \mathrm{D}$ wavelength demultiplexing [1]. A structure generating different spatial dispersions for $\mathrm{x}$ and $\mathrm{y}$ axis respectively, with novel employment of Free Spectral Range (FSR), enables $2 \mathrm{D}$ wavelength demultiplexing. The concept has been employed to an arrayed waveguide device and thorough analysis has been reported [2]. However, the structure requires stacking of many layers making the method impractical for fabrication. In this report we propose a device using only a single arrayed waveguide device in a simple planar structure [3].

\section{Device configuration}

The device we propose (Fig. 1) is the planar waveguide version of the device we reported in 2D structure [1]. Instead of using a free space light beam, we use guided light in a planar waveguide propagated in appropriate direction for generating required light path difference by a waveguide array [3]. A lens not shown in Fig. 1 is required to focus the demultiplexed light beam into output ports. The 3D structure in Ref. 1 generates discrete light focusing spots for each phase matching wavelength, but in 2D structure, the spot is scanned along a line folded back at the end of a FSR just like a deflected electron beam in the cathode ray tube (CRT) for a television.

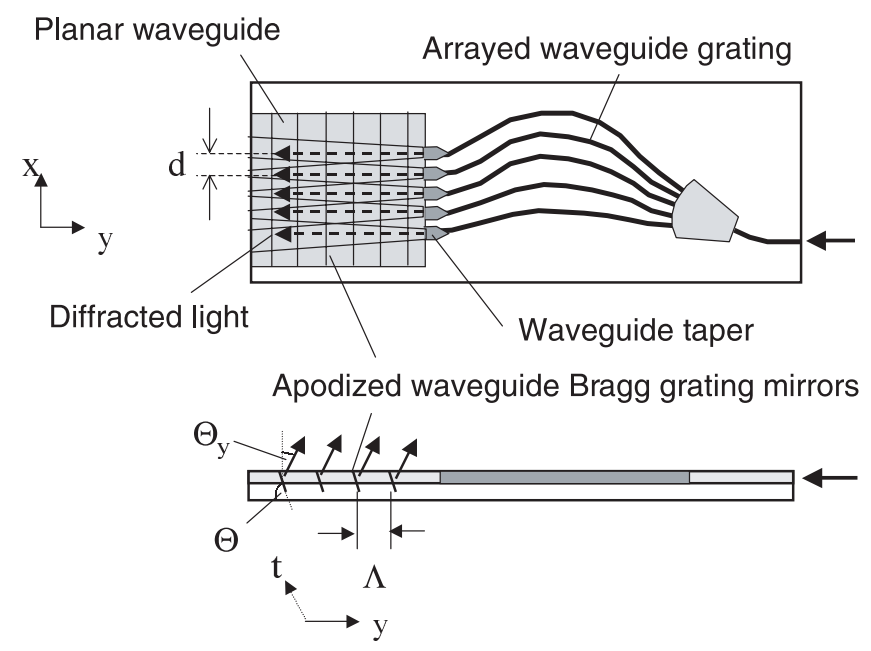

Fig. 1. Planar arrayed waveguide device structure for 2D wavelength demultiplexing.

In the $2 \mathrm{D}$ demultiplexing method, the beam spot position shift in the $\mathrm{x}$ axis for different wavelengths can be larger than that for the y axis. To realize a 2D wavelength demultiplexing, several wavelengths should be directed into a single $\mathrm{x}$ (and $\mathrm{y}$ ) coordinate. The multiple FSR is used for this purpose. The $2 \mathrm{D}$ wavelength-demultiplexing device requires a section that generates large spatial dispersion associated with FSR and a section with smaller spatial dispersion. An arrayed waveguide grating (AWG) [4] is connected to a planar waveguide grating structure for creating a virtual image 2D aperture array 
(Fig. 2). The AWG generates a large dispersion in the $\mathrm{x}$ axis. The 2D aperture array generates smaller dispersion in the y axis. Many types of grating structures such as UV induced grating or surface grating $[5,6]$ can be used as the partial reflecting mirrors. The bulk holographic grating or even a conventional reflection grating can also be used as a $2 \mathrm{D}$ aperture generator.

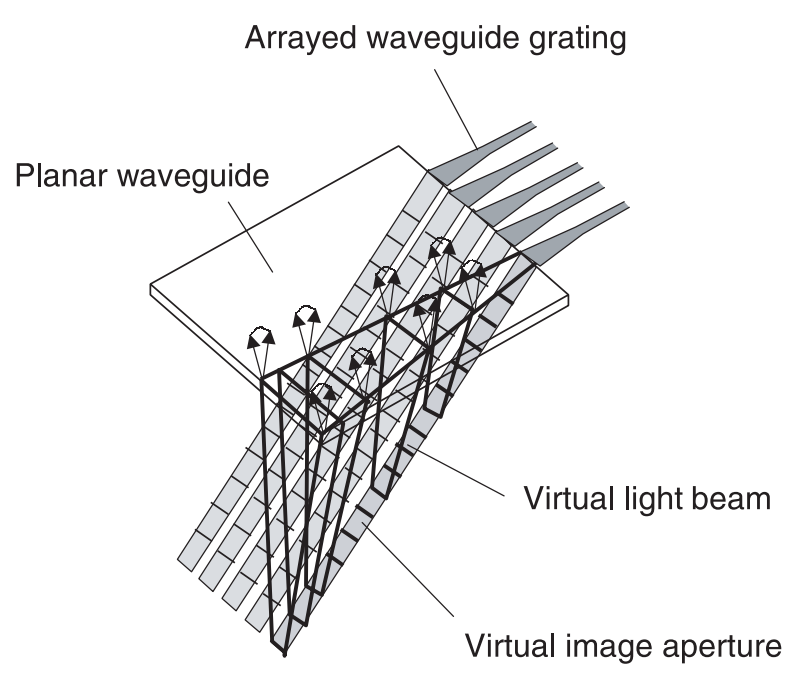

Fig. 2. Virtual 2D light aperture array generated by planar waveguide Bragg grating.

\section{Filter response}

We obtain the total light field I as a function of beam direction in $\mathrm{x}$ and $\mathrm{y}$ axis $\left(\Theta_{\mathrm{x}}\right.$ and $\left.\Theta_{\mathrm{y}}\right)$, by summing the light field from each aperture over the waveguide array number $\mathrm{r}$ and grating number $\mathrm{q}$.

$$
\begin{aligned}
\mathrm{I}= & \sum \mathrm{A}_{\mathrm{qr}} \exp \left[\mathrm{jkr}\left(-\mathrm{d} \sin \Theta_{\mathrm{x}}+\mathrm{DL}_{\mathrm{a}}\right)\right] \exp \left[\mathrm{jkq} \Lambda\left(\sin \Theta_{\mathrm{y}}+1\right) \cos \Theta_{\mathrm{x}}\right] \\
& \times \iint \mathrm{B}(\mathrm{x}, \mathrm{t}) \exp \left[\mathrm { jk } \left\{-\mathrm{x} \sin \Theta_{\mathrm{x}}+\mathrm{t} \cos \Theta_{\mathrm{x}}\left[-\cos \left(\Theta_{\mathrm{y}}-\Theta+\pi / 2\right)\right.\right.\right. \\
& +\cos (\Theta)]\}] \mathrm{dxdt}
\end{aligned}
$$

where $\mathrm{k}$ is the light wave number, $\mathrm{t}$ is the coordinate on a grating mirror, $\Theta$ is the mirror angle, $d$ is the waveguide output separation of $A W G$ and $\mathrm{DL}_{\mathrm{a}}$ is the adjacent path length difference in arrayed waveguide. The grating period is $\Lambda$.

When the light power $\mathrm{A}_{\mathrm{qr}}$ is equally divided among $2 \mathrm{D}$ aperture array and rectangular light power distribution $\mathrm{B}(\mathrm{x}, \mathrm{t})$ in each aperture is assumed, the light field becomes,

$$
\begin{aligned}
& \mathrm{I}=\mathrm{A}\left\{\sin \left[\mathrm{kN}_{\mathrm{w}}\left(-\mathrm{d} \sin \Theta_{\mathrm{x}}+\mathrm{DL}_{\mathrm{a}}\right) / 2\right] \sin \left[\mathrm{kM}_{\mathrm{w}} \Lambda\left(\sin \Theta_{\mathrm{y}}+1\right) \cos \Theta_{\mathrm{x}}\right] / 2\right. \\
& \quad /\left\{\sin \left[\mathrm{k}\left(-\mathrm{d} \sin \Theta_{\mathrm{x}}+\mathrm{DL}_{\mathrm{a}}\right) / 2\right] \sin \left[\mathrm{k} \Lambda\left(\sin \Theta_{\mathrm{y}}+1\right) \cos \Theta_{\mathrm{x}} / 2\right]\right\} \\
& \quad \times \sin \left(-\mathrm{kS} \sin \Theta_{\mathrm{x}} / 2\right) \sin \left[\mathrm{kT}\left[-\cos \left(\Theta_{\mathrm{y}}-\Theta+\pi / 2\right)+\cos (\Theta)\right] \cos \Theta_{\mathrm{x}} / 2\right] \\
& /\left\{\left(-\mathrm{kS} \sin \Theta_{\mathrm{x}} / 2\right)\left[\mathrm{kT}\left[-\cos \left(\Theta_{\mathrm{y}}-\Theta+\pi / 2\right)+\cos (\Theta)\right] \cos \Theta_{\mathrm{x}} / 2\right]\right\}
\end{aligned}
$$


where $\mathrm{S}$ is the aperture width at the end of the waveguide array and $\mathrm{T}$ is the cross sectional length of the slanted grating aperture. $\mathrm{N}_{\mathrm{w}}$ is the number of waveguides in AWG and $M_{w}$ is the number of grating along a light path. The maximum output is centred on $\Theta_{y}=2 \Theta-\pi / 2$. The mirror angle $\Theta$ should be tilted in accordance with $\Theta_{\mathrm{y}}$ designed to attain desired performance. The output becomes the same as that of the conventional AWG for each spatial axis.

The beam steering angle change caused by wavelength shift $\Delta \lambda$ in $\mathrm{x}$ axis $\Delta \Theta_{\mathrm{x}}$ and $\mathrm{y}$ axis $\Delta \Theta_{\mathrm{y}}$ are derived as $\Delta \Theta_{\mathrm{x}}=-\mathrm{S}_{\mathrm{x}} \Delta \lambda /(\mathrm{nd})$ and $\Delta \Theta_{\mathrm{y}}=$ $\mathrm{S}_{\mathrm{y}} \Delta \lambda /\left(\mathrm{n} \Lambda \cos \Theta_{\mathrm{x}} \cos \Theta_{\mathrm{y}}\right)$ respectively with $\mathrm{n}$ being the refractive index. Here $\mathrm{S}_{\mathrm{x}}$ and $\mathrm{S}_{\mathrm{y}}$ are interference order for $\mathrm{x}$ axis and $\mathrm{y}$ axis respectively. The half width of the filter response peak is defined by $\Delta \lambda_{\mathrm{x}}=\lambda /\left(\mathrm{N}_{\mathrm{w}} \mathrm{S}_{\mathrm{x}}\right)$ for the $\mathrm{x}$ axis which is narrower than that for the y axis $\Delta \lambda_{\mathrm{y}}=\lambda /\left(\mathrm{M}_{\mathrm{w}} \mathrm{S}_{\mathrm{y}}\right)$.

\section{Design procedure}

The device is designed using the filtering performance deduced from Eqs. 1 and 2. We consider a device demultiplexing NM wavelengths into NxM array of light beams. $\mathrm{N}$ is equal to the resolvable wavelength number in a FSR along the $\mathrm{x}$ axis and $\mathrm{M}$ the resolvable wavelength number along the $\mathrm{y}$ axis. A condition $\mathrm{N}_{\mathrm{w}}>2 \mathrm{~N}$ and $\mathrm{M}_{\mathrm{w}}>2 \mathrm{M}$ is required to avoid the overlap between light beams.

The interference order, $S_{x}$ for $x$ axis, at centre wavelength is determined from wavelength resolution and number of resolvable wavelengths in FSR. The interference order for $\mathrm{y}$ axis is determined from the total wavelength scan range $\mathrm{D} \lambda_{\mathrm{sc}}$. These lead to $\mathrm{S}_{\mathrm{x}}=\left[\lambda_{0} /\left(\mathrm{D} \lambda_{\mathrm{sc}} / \mathrm{M}\right)\right]$ and $\mathrm{S}_{\mathrm{y}}<\left(\lambda_{0} / \mathrm{D} \lambda_{\mathrm{sc}}\right)$ where $\lambda_{0}$ is the centre wavelength. For the first order grating $\mathrm{S}_{\mathrm{y}}=1$.

\subsection{AWG}

The array spacing $d$ is defined from the required beam divergence angle $\mathrm{D} \Theta_{\mathrm{x}}$ along the $\mathrm{x}$ axis. With $\mathrm{D} \Theta_{\mathrm{sc}}$ being the angle scan range of the light beam for $2 \mathrm{D}$ demultiplexing, $\mathrm{D} \Theta_{\mathrm{x}}=\mathrm{D} \Theta_{\mathrm{sc}} / \mathrm{N}$. The angle $\mathrm{D} \Theta_{\mathrm{sc}}$ is limited by the light divergence angle of each aperture. The array spacing is obtained by $\mathrm{d}=2 \lambda_{0} /\left(\mathrm{N}_{\mathrm{w}} \mathrm{nD} \Theta_{\mathrm{x}}\right)$. The length difference $\mathrm{DL}_{\mathrm{a}}$ is defined by beam direction along $\mathrm{x}$ axis $\Theta_{\mathrm{xa}}$ at an anchor wavelength $\lambda_{\mathrm{a}}$ and $\mathrm{S}_{\mathrm{x}}$. So that, $\mathrm{DL} \mathrm{a}=\left(\mathrm{S}_{\mathrm{x}} \lambda_{\mathrm{a}}-\right.$ $\left.\Theta_{x a} n d\right) / n_{a}$, where $n_{a}$ is the effective refractive index of the waveguide array.

\subsection{Grating}

The light beam angle shift along y axis, induced by wavelength shift of a FSR, should be larger than divergence angle of the light beam along y axis $\mathrm{D} \Theta_{\mathrm{y}}$. This restriction defines the number of grating $M_{w}$. The number of grating is calculated by $M_{w}=2 S_{x} / S_{y}$. We make the beam divergence angle along the $\mathrm{x}$ and $\mathrm{y}$ axis $\left(\mathrm{D} \Theta_{\mathrm{x}}\right.$ and $\left.\mathrm{D} \Theta_{\mathrm{y}}\right)$ equal, so that the light beam has circular cross section. This sets the diffraction direction $\Theta_{\mathrm{y} 0}$ at center wavelength as $\cos \Theta_{\mathrm{y} 0}=2 /\left[\mathrm{S}_{\mathrm{x}} \mathrm{D} \Theta_{\mathrm{x}}+1 /\left(\mathrm{S}_{\mathrm{x}} \mathrm{D} \Theta_{\mathrm{x}}\right)\right]$. Finally, the grating period $\Lambda$ is defined by beam direction along y axis $\Theta_{\text {ya }}$ at an anchor wavelength $\lambda_{\mathrm{a}}$ and $\mathrm{S}_{\mathrm{y}}$. Thus, 
$\Lambda=\mathrm{S}_{\mathrm{y}} \lambda_{\mathrm{a}} /\left[\mathrm{n}_{\mathrm{g}}\left(\sin \Theta_{\mathrm{ya}}+1\right) \cos \Theta_{\mathrm{ya}}\right]$ where $\mathrm{n}_{\mathrm{g}}$ is the refractive index of the grating portion. The beam angle difference between those for the anchor and center wavelength is calculated by using equations for $\Delta \Theta_{\mathrm{x}}$ and $\Delta \Theta_{\mathrm{y}}$ described in section 3 .

The angle $\Theta_{\mathrm{y} 0}$ limits the practical design range. For easiness of fabrication, $\Theta_{\mathrm{y} 0}<1.2 \mathrm{rad}$ is desireable, which sets an upper limit for parameter $\mathrm{S}_{\mathrm{x}} \mathrm{D} \Theta_{\mathrm{x}}=(\mathrm{M} / \mathrm{N})\left[\mathrm{D} \Theta_{\mathrm{sc}} /\left(\mathrm{D} \lambda_{\mathrm{sc}} / \lambda_{0}\right)\right]$ to be less than 4 . When the device is designed for a narrow wavelength range, the light beam angle scan range should be also narrow accordingly.

\section{Simulation}

Example of calculated 2D light spot array for wavelength channels is shown in Fig. 3. The wavelength ranges from 1.52 to $1.58 \mu \mathrm{m}$. Corresponding interference order for $\mathrm{x}$ axis is from $\mathrm{S}_{\mathrm{x}}=405$ to 390 and $\mathrm{S}_{\mathrm{y}}=1$ for $\mathrm{y}$ axis. The AWG portion has 32 waveguide array; path length difference $\mathrm{DL}_{\mathrm{a}}$ of $616.4 \mu \mathrm{m}$ and output port separation $\mathrm{d}=9.3 \mu \mathrm{m}$. The grating portion has grating with 370 mirrors with period $\Lambda$ of $0.803 \mu \mathrm{m}$. The light beam divergence angle generated by $2 \mathrm{D}$ aperture array was $0.01 \mathrm{rad}$. A grating fabricated with refractive index change of $\mathrm{dn} \sim 10^{-3}$ is required to reflect sufficient light out of the planar waveguide to attain $1 \mathrm{~dB}$ loss, which is approximately estimated by $\mathrm{dn}=\mathrm{n}_{\mathrm{g}}\left[(1-\mathrm{R})^{\left.-1 / 2 \mathrm{M}_{\mathrm{w}}-1\right] / 2}\right.$ where $\mathrm{R}$ is the total fraction of the reflected light power. The required refractive index change becomes lower for narrower wavelength range and larger wavelength channels due to the increase in the number of mirrors.

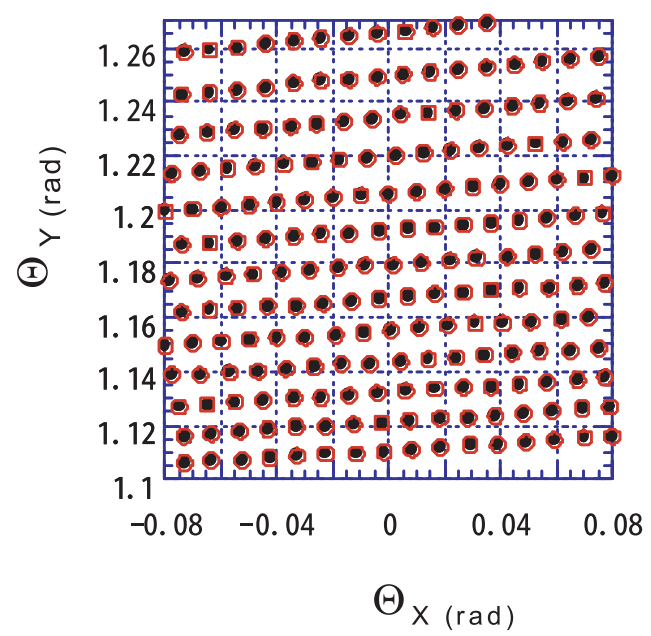

Fig. 3. Simulated light spot center of $2 \mathrm{D}$ wavelength demultiplexing from 1520 to $1580 \mathrm{~nm}$ wavelength.

Since the dispersion in y axis is a function of angle $\Theta_{\mathrm{y}}$ the light spot interval shows a angle dependence in this direction. The light beam shape and filter response peak shape remains the same through out the wavelength range as revealed by simulation. 
The chip size for high index contrast waveguide is approximately 1 square centimeter in which the grating portion occupies an area of 0.3 square millimeter. The distance between chip (lens) and focal plane is $5 \mathrm{~mm}$ assuming $0.05 \mathrm{~mm}$ demultiplexed light spot separation.

\section{Conclusion}

Simple arrayed waveguide grating structure that demultiplexes wavelengths to output ports arranged in $2 \mathrm{D}$ plane have been reported. The planar arrangement enables easy fabrication. An AWG is connected to a grating device forming a virtual image 2D aperture array, which generates smaller dispersion in different direction than that of AWG. The 2D wavelength demultiplexing was verified by calculation. Design parameters such as the waveguide mirror angle and spacing between mirrors were calculated. The design parameters were in reasonable value range for fabrication to obtain a device with several-hundred-wavelength channels. 